\title{
¿MIRAMOS EL MUNDO A TRAVÉS DE LA GRIETA? ANÁLISIS DEL TRATAMIENTO EDITORIAL DE LA CUMBRE INTERCOREANA DESDE ARGENTINA
}

\author{
Do we look at the world through the crack? Analysis of the editorial
} treatment of the inter-Korean summit from Argentina

\section{Geraldina Dana*, Catalina Mas"*, María Emilia Reiszer*** y Emilio Gabriel Soto ${ }^{* * * *}$}

\section{RESUMEN}

El 27 de abril del 2018 se realizó la primera reunión en once años entre los mandatarios de Corea del Norte y Corea del Sur. Este trabajo aborda el tratamiento editorial de los periódicos argentinos Clarín y Página 12 sobre dicha cumbre. El objetivo es observar si hay diferencias en la frecuencia de cobertura y el tratamiento editorial que estos medios le profieren. Se intentó explicar esto a través de las teorías del Framing, Agendasetting e Intermedia Agenda-setting, con base en autores como Kiousis (2004), Zunino (2016), Martini y Luchessi (2004), Amadeo (2008), Aruguete y Amadeo (2013) y Aruguete (2015). Dado que dicha cumbre no es controversial en la Argentina, la hipótesis refirió a que estos periódicos, si bien divergentes en la arena nacional, tuvieron una cobertura similar en este caso. A tal fin, se realizó un relevamiento de carácter mixto "de etapas sucesivas" (cuantitativo y posteriormente cualitativo), de la cobertura de ambos medios el cual se procesó, codificó y construyó un modelo de medición mediante diversas matrices de datos. El análisis de los resultados del índice indica que la relevancia dada por ambos diarios fue media, con la aparición de notas de tapa y contratapa. En cuanto al tratamiento editorial, ambos periódicos se enfocaron en conceptos como la desnuclearización y el análisis de las consecuencias geopolíticas. Sin embargo, Clarín lo hizo con inclinaciones en favor de la posición de Corea del Sur,

\footnotetext{
* Investigadora en Instituto de Investigaciones Gino Germani, Licenciada en Ciencia Política, Universidad de Buenos Aires, Buenos Aires, Argentina. Correo electrónico: geraldinadanag@gmail.com

** Estudiante de Ciencia Política, Universidad de Buenos Aires, Buenos Aires, Argentina. Correo electrónico: catalina.mas22@gmail.com

*** Estudiante de Ciencia Política, Universidad de Buenos Aires, Buenos Aires, Argentina. Correo electrónico: emi.reiszer@gmail.com

***** Estudiante de Ciencia Política, Universidad de Buenos Aires, Buenos Aires, Argentina. Correo electrónico: emiliosoto96@hotmail.com
} 
¿Miramos el mundo a través de la grieta? Análisis del tratamiento editorial de la cumbre intercoreana desde Argentina

sumado a la presencia de recursos retóricos de crítica hacia el líder y gobierno de Corea del Norte. Por este motivo, la hipótesis se cumple sólo parcialmente.

Palabras claves: Clarín, Página 12, Corea, cumbre intercoreana, Argentina.

RECIBIDO: Febrero 2019

ACEPTADO: Agosto 2019

\section{ABSTRACT}

On April 27th 2018, the first summit in eleven years was held between North Korean and South Korean chief executives. This paper addresses Argentinian newspapers Clarín and Página 12 editorial approaches pertaining to that summit. The purpose is to analyze whether there is a difference in the frequency of the media coverage and the editorial approach of both newspapers. This paper tries to explain these phenomena through Framing, Agenda-setting and Intermedia Agenda-setting theories, based upon authors such as Kiousis (2004), Zunino (2016), Martini, Luchessi (2004), Amadeo (2008), Amadeo and Aruguete (2013) and Aruguete (2015). Given that the summit is not considered to be controversial in Argentina, the hypothesis assumes that these newspapers, although divergent within the national arena, will have a similar coverage in this case. Following this aim, a mixed gathering "of successive stages" was done (first, a quantitative one, and then, a qualitative one) of media coverage on both newspapers. This was then processed and codified in order to build a measurement model utilizing different data matrices. The analysis of the index results show that the relevance given by both newspapers was medium level, with the presence of cover and back cover articles. When it comes to the editorial approach, both papers focused on concepts such as de-nuclearization and analysis of geopolitical consequences therein. However, Clarín adopts a bias position favoring the South Korean stand, together with rhetorical resources criticizing North Korean leadership and government. Thus, for this reason, the hypothesis has only a partial corroboration.

Key words: Clarín, Página 12, Korea, Inter-korean summit, Argentina.

\section{Introducción}

El presente trabajo aborda el tratamiento editorial de los periódicos argentinos Clarín y Página 12 respecto a la cumbre llevada a cabo el 27 de abril de 2018 entre la República de Corea (Corea del Sur) y la República Popular Democrática de Corea (Corea del Norte), separadas desde 1945 y en guerra a partir de 1950, con un armisticio firmado en 1953. Previo a la cumbre de principios del año 2018 sólo se habían celebrado 
dos cumbres intercoreanas, en los años 2000 y 2007. La del 2018 se llevó a cabo en la Casa de la Paz de la Zona de Seguridad Conjunta.

Aunque no estaba en perspectiva la unificación de la península, el objeto principal de la cumbre fue iniciar las negociaciones para un tratado de paz. Los puntos de acuerdo de la reunión fueron: la detención de las hostilidades recíprocas, la suspensión de las emisiones de propaganda en la frontera, el mantenimiento de conversaciones regulares de alto nivel militar y político, la resolución de las diferencias sobre la frontera marítima para evitar enfrentamientos con pescadores, la reanudación de las reuniones entre familiares divididos como consecuencia de la guerra y, especialmente la desnuclearización total de la península (Clarín, 28/04/2018).

Como información a considerar, ninguno de los dos Estados coreanos es socio estratégico para la Argentina. De hecho, el país sudamericano y Corea del Norte no mantienen actualmente relaciones diplomáticas, como tampoco vínculos institucionales ni económicos. No obstante existieron relaciones diplomáticas entre junio de 1973 y junio de 1977 , los intentos de apertura de oficinas comerciales sin estatus diplomático en Buenos Aires a finales de los años noventa fracasaron.

En cambio, Argentina y Corea del Sur iniciaron relaciones diplomáticas bilaterales en 1962, que permanecen inalterables desde entonces. Desde 1962 el Estado argentino reconoce a Corea del Sur como representante exclusivo del Estado coreano, posicionamiento que mantiene hasta la actualidad (Oviedo, 2016, p.123, destacado propio). La única excepción la constituye el período abarcado entre 1973 - 1977. Siguiendo a Oviedo $\left(2016\right.$, p.122) ${ }^{1}$, las relaciones entre Argentina y Corea del Sur

$1 \mathrm{El}$ autor realiza una categorización con respecto a las relaciones bilaterales entre Estados, las cuales pueden ser: relaciones sin explotar, subexplotadas, explotadas intensamente, plenamente explotadas y sobreexplotadas. Entre ellas el punto de equilibrio lo constituyen las relaciones plenamente explotadas. 
¿Miramos el mundo a través de la grieta? Análisis del tratamiento editorial de la cumbre intercoreana desde Argentina

pueden ser definidas como "subexplotadas". Por añadidura, respecto a las relaciones económicas, el comercio bilateral registrado en el año 2016 entre ambos Estados arrojó un total de 1.628 millones de dólares, lo que para el comercio argentino simboliza un $1,4 \%$ del volumen total de importaciones y exportaciones de dicho año (cálculos propios en base a datos del INDEC). Estos datos permiten afirmar que, si bien existen relaciones políticas entre Argentina y la República de Corea, así como vínculos comerciales, estos no son ampliamente significativos para el país sudamericano.

La primera pregunta de la presente investigación apunta al grado de relevancia que dos periódicos: Clarín y Página 12 le brindaron a la histórica cumbre entre las dos Coreas, considerando su lejanía geográfica y cultural, y su escasa significación económica para la República Argentina. En este sentido se utilizan la teoría de la Agenda-setting y las variables cuantitativas que dan cuenta de la atención puesta al acontecimiento (frecuencia de la cobertura) y de la prominencia en relación jerárquica con otros temas. Asimismo, la investigación se basa en los presupuestos de recortes de la realidad que son abordados por el primer momento de la teoría del Framing en tanto "hechos críticos" (Pride citado en Amadeo, 2008, p.188).

La selección de los periódicos responde a dos criterios: en primer lugar, a la circulación nacional de ambos y, en segundo lugar, a sus diferentes líneas editoriales. Clarín es el principal diario argentino en cuanto a su circulación, poseyendo un volumen de tirada media de 133.827 ejemplares de lunes a sábado, con un pico los domingos de 400.919 ejemplares (IVC, 2018). Además, el diario acapara aproximadamente el 40\% de la cobertura publicitaria de la industria (Becerra y Mastrini citado en Zunino, 2016, p.136). Según Martini y Luchessi (2004), el diario Clarín duplica la tirada del segundo en preferencia del electorado, La Nación, y suma más que todos los demás diarios juntos. En términos de su audiencia, Clarín apunta al lector promedio (Martini y Luchessi, 2004). En cuanto a su línea editorial, Clarín se caracterizó por una clara oposición a los gobiernos kirchneristas (2003-2015), y una mirada más amigable hacia el actual gobierno de Cambiemos (desde 2015 a la actualidad). En cambio, Página 12 
presenta una tirada promedio de 9.923 ejemplares de lunes a sábado, llegando a 25.054 ejemplares para el domingo. Este periódico, más pequeño y con un público más selecto -de clase media-alta, con altos niveles educativos (Zunino, 2016)- fue favorable, en términos editoriales, a los gobiernos kirchneristas y actualmente posee una mirada crítica del Gobierno Nacional. Estas diferencias permitirán llevar a cabo un análisis comparativo entre dos medios marcadamente diferentes y particularmente importantes para las dos opiniones políticas predominantes en Argentina. Una de ellas, referenciada con la línea editorial de Página 12, afín a los gobiernos kirchneristas, y a los sectores de izquierda dentro del peronismo. La otra, asimilable al lineamiento editorial de Clarín, desfavorable al peronismo en general, y al kirchnerismo en particular. La tajante oposición entre estas dos posturas políticas se conoce en el debate público argentino como "la grieta".

En este sentido, la segunda pregunta que guía la presente investigación apunta al tratamiento editorial proferido por ambos periódicos a la cumbre intercoreana del 27 de abril de 2018. Aquí se utiliza la metodología cualitativa a fin de descifrar la valencia como la tercera dimensión de la relevancia, teorizada por Kiousis (2004), que da cuenta de la presencia o ausencia de elementos afectivos. Asimismo, se complementa con los análisis que abordan el segundo momento de la teoría de Framing, que trata la producción del texto y los atributos subyacentes.

La hipótesis que orienta este trabajo es que, al tratarse de dos Estados que no son aliados comerciales estratégicos de la Argentina, y que se encuentran lejos en términos culturales y geográficos de la misma, dos periódicos de marcadas diferencias editoriales a nivel nacional no harán eco de sus divergencias ideológicas a la hora de informar acerca de este evento de política internacional. Esta hipótesis, estaría sustentada por los desarrollos teóricos inscriptos en la teoría de la Intermedia Agendasetting, ya que este suceso mediático tendría cobertura ante la presión internacional, 
¿Miramos el mundo a través de la grieta? Análisis del tratamiento editorial de la cumbre intercoreana desde Argentina

pero los periódicos no adoptarían una postura propia sino homogeneizarían el discurso con diarios de mayor tirada a nivel internacional.

\section{¿Miramos el mundo a través de la grieta?}

\section{Metodología}

El presente trabajo se sirvió de una estrategia metodológica mixta. En particular, realiza el tipo de integración metodológica que Gallart (1992) llama "de etapas sucesivas", donde se parte de un diagnóstico cuantitativo, con el objeto de caracterizar el fenómeno en estudio, y se adentra en un análisis cualitativo del mismo, para comprenderlo.

En este sentido, se realizó un relevamiento bibliográfico de trabajos acerca de agenda-setting y framing (Kiousis, 2004; Martini y Luchessi, 2004; Amadeo, 2008; Aruguete y Amadeo, 2013; Aruguete, 2015; Zunino, 2016), en una primera instancia. Asimismo, se consultaron recursos en línea para complementar el contexto histórico y la información estadística que esta investigación toma como base (INDEC, 2014; IVC, 2018).

Como Gallart (1992) aconseja, se buscó llegar a una clara relación entre cada uno de los interrogantes de la investigación y la estrategia metodológica utilizada para contestar en cada caso. De allí que la primera pregunta de investigación, concerniente a la relevancia dada por cada uno de los periódicos a la cumbre intercoreana, se responda a partir de información cuantitativa. En cambio, el segundo interrogante, referido al tratamiento editorial del tema por parte de cada diario, es abordado a través de información cualitativa, ya que, según la autora, ésta es la más adecuada para realizar análisis comparativos.

Por todo lo anterior, en un segundo momento, se relevaron 30 (treinta) ejemplares físicos de los dos periódicos de circulación nacional escogidos, Clarín y Página 12. El relevamiento fue realizado entre el 23/04/2018, cinco días antes de la cumbre intercoreana, y el 08/05/2018. De esta manera, 15 (quince) unidades de análisis 
corresponden a los ejemplares de Clarín en estas fechas, y otras 15 (quince), a los de Página 12. Con base en estas unidades de análisis, se construyó una primera matriz de datos cuantitativos. En la misma, se tomaron en cuenta las siguientes variables para caracterizar a las unidades de análisis: fecha, periódico, día de la semana, número total de páginas, número de páginas de la sección internacional, porcentaje que esta sección ocupa en ese ejemplar, cantidad total de notas sobre la cumbre intercoreana y tirada promedio para ese día de la semana de ese diario. La cuantificación porcentual de la sección internacional respondió a la búsqueda de comparar en términos relativos ambos periódicos, ya que Clarín tiene una extensión de casi dos veces más de Página 12. Además de la cantidad de notas sobre la cumbre intercoreana, se tomó el día de la semana en que fueron publicadas como un indicador de relevancia, considerando que ambos periódicos tienen una tirada más extensa los fines de semana, y, especialmente, los días domingo.

En tercer lugar, se ubicaron las piezas periodísticas referentes a la temática publicadas por ambos periódicos, obteniendo así una muestra intencional formada por 21 (veintiún) notas en total: 6 (seis) correspondientes a Página 12 y 15 (quince) en el diario Clarín. Con base en las mismas se confeccionó otra matriz de datos cuantitativos. La misma detalla si aparecen o no en la portada o la contratapa, su ubicación en la página (mitad inferior o superior), su número de página, si ésta es par o impar, si está o no firmada, si tiene bajada o entradilla, si tiene una valencia explícitamente definida o no, si abre sección o es una nota de opinión, su cantidad de palabras y su tamaño según ésta, el tamaño del título (más o menos de la mitad del ancho de la página), la superficie absoluta de la pieza en centímetros cuadrados y su tamaño según ésta, y si contiene o no imágenes. Siguiendo las dimensiones que componen el índice de importancia de Zunino (2016), una nota es más relevante para un diario si: aparece en la portada o la contratapa, está en la mitad superior de la página, se ubica en una página impar, está firmada, tiene bajada, tiene una valencia explícitamente definida, abre sección, es una 
¿Miramos el mundo a través de la grieta? Análisis del tratamiento editorial de la cumbre intercoreana desde Argentina

nota de opinión, es más grande -en cualquiera de las tres formas del tamaño que aquí se evaluaron- y contiene imágenes. De esta manera, los datos de las primeras dos matrices nos permiten explicar cuantitativamente la relevancia.

Se enumeraron los 30 (treinta) periódicos estudiados. Por un lado, se le asignó un número según el diario (impares para Clarín y pares para Página 12) y la fecha del mismo (fijando como unidad número 1 al diario Clarín del día 24 de abril del 2018 y como unidad número 30, y última, al diario Página 12 del día 8 de mayo del 2018). Asimismo, se procedió a subdividir la numeración de los diarios donde apareciera más de una nota -por ejemplo, la U8.1 corresponde a la primera nota que aparece en el ejemplar correspondiente a la cuarta fecha de Página 12, mientras que la U8.2 corresponde a la segunda nota que aparece en ese mismo diario-. De esa forma se puede identificar de manera clara cada nota tomada para el análisis. Nuestras unidades de análisis están numeradas, entonces, a partir de la U5.1 debido a que no se presentan notas sobre la temática estudiada en los números anteriores de Clarín y Página 12. La lista completa de referencias de las notas relevadas con su correspondiente numeración consta en la tabla 1.

Tabla 1. Detalle de notas analizadas dentro de las unidades de análisis

U5.1: “'Es muy honorable', el extraño elogio al dictador norcoreano”, Clarín, 25 de abril de 2018.

U8.1: "La guerra que no terminó", Página 12, 26 de abril de 2018.

U8.2: "Cumbre histórica de las Coreas", Página 12, 26 de abril de 2018.

U9.1: "Histórica cumbre entre las dos Coreas, el prólogo de la reunión de Kim con Trump". Clarín, 27 de abril de 2018.

U9.2: "Cada detalle de la cumbre coreana representa un símbolo protocolar", Clarín, 27 de abril de 2018.

U10.1: Julián Varsavsky, "La nación bifronte vuelve a mirarse", Página 12, 27 de abril de 2018.

U10.2: "Kim y Moon se dieron la mano", Página 12, 27 de abril de 2018. 
U11.1: Cantelmi Marcelo, "Las Coreas, Irán, Trump, Macron: de capacidades y posibilidades, Clarín, 28 de abril de 2018.

U11.2: "Las dos Coreas acuerdan profundizar los vínculos y desnuclearizar la península", Clarín, 28 de abril de 2018.

U11.3: "Las diferencias que encierra el término desnuclearización", Clarín, 28 de abril de 2018.

U11.4: Erlich, "Tira y afloja", Clarín, 28 de abril de 2018.

U12.1: “Todos felices con la cumbre de las Coreas”, Página 12, 28 de abril de 2018.

U13.1: "La propaganda norcoreana celebra la histórica cumbre con el sur", Clarín, 29 de abril de 2018.

U13.2: "EE. UU. ultima los detalles para la cita con Kim”, Clarín, 29 de abril de 2018.

U15.1: "Corea del Norte ofrece desmantelar un centro de ensayos nucleares", Clarín, 30 de abril de 2018.

U15.2: "La planta de Punggye-ri, en las montañas del noreste del país", Clarín, 30 de abril de 2018.

U17.1: "La cumbre entre Trump y Kim podría ser en la frontera coreana", Clarín, 2 de mayo de 2018.

U18.1: Boron Atilio, "Cuba, Trump y el diálogo coreano", Página 12, 2 de mayo de 2018.

U21.1: "Diplomacia del Ping Pong: las dos Coreas se acuerdan en el Mundial de Suecia", Clarín, 4 de mayo de 2018.

U25.1: Lagos Ricardo, "Vigencia de la disuasión nuclear", Clarín, 6 de mayo de 2018.

U27.1: “Corea del Norte acusa a EE. UU de provocación”, Clarín, 7 de mayo de 2018.

Fuente: Elaboración propia

Los datos de ambas matrices cuantitativas fueron codificados. Luego, se confeccionó a los efectos del presente trabajo un índice de importancia, cuyo valor 
¿Miramos el mundo a través de la grieta? Análisis del tratamiento editorial de la cumbre intercoreana desde Argentina

máximo posible es 18 (dieciocho) y su valor mínimo posible es 1 (uno). A partir del mismo, las noticias se clasificaron según un criterio aritmético en los siguientes tres grupos principales, que a su vez se subdividen aritméticamente en tres subgrupos para cada categoría:

1 a 6 puntos = importancia baja $(1$ y 2 : baja inferior; 3 y 4 : baja; 5

y 6: baja superior).

7 a 12 puntos = importancia media ( 7 y 8 : media inferior; 9 y 10 :

media; 11 y 12: media superior).

13 a 18 puntos= importancia alta. (13 y 14: alta inferior; 15 y 16 :

alta; 17 y 18: alta superior).

Tabla 2. Libro de códigos

1. Día de la semana en que apareció la nota: Se computa 1 si apareció en un día hábil; 2 un sábado y 3 si apareció un domingo.

2. Aparición en portada o en contratapa: La variable toma un valor de 1 si apareció en la tapa o la contratapa, y de 0 si no lo hizo.

3. Ubicación: Se le asigna un valor de 1 si se encuentra en la mitad superior de la página, y de 0 si se sitúa en la mitad inferior.

4. Firma: Si la nota se encuentra firmada por un/a autor/a, toma el valor de 1. Si ésta no posee firma o es recogida de una agencia de noticias, se le asigna un 0.

5. Bajada o entradilla: Se le asigna un 1 si la posee, y un 0 si no.

6. Valencia: Si la nota posee una valencia explícitamente definida (sea positiva o negativa), se califica con un 1 . Si no, con 0 .

7. Apertura de sección o nota de opinión: Si la nota responde positivamente a alguna de esas dos condiciones, se califica con 1 . En caso contrario, con $0 .^{2}$

2 En un caso (Clarín, 28/04/2018) apareció un cómic de humor político. Se lo tomó como equivalente a una nota de opinión a los fines de la presente codificación, y por eso se lo calificó con 1. 
8. Tipo de página: Si el artículo aparece en la página impar, se le asigna un 1 . Si no, un 0.

9. Tamaño por cantidad de palabras: Si es "grande", se le asigna un 3; si es "mediana", un 2, y si es "chica", un 1.

10. Tamaño del título: Si éste ocupa la mitad de la página o más, se califica con 1 . Si no, con 0 .

11. Tamaño por superficie: Si ésta es "grande", se le asigna un 3; se califica con 2 si es "mediana", y con 1 si es "chica".

12. Acompañamiento de elementos gráficos: Si posee imágenes o fotografías, se le asigna un 1 . Si no las posee, un 0.

Fuente: Elaboración propia

En cuarto lugar, se construyó una matriz de datos cualitativos de la totalidad de las notas con la finalidad de contestar nuestro segundo interrogante, relativo a las líneas editoriales. En la misma se incluyeron las siguientes variables: título, nombre del autor o agencia si los hubiere, sección en la que se encuentra, valencia (positiva, negativa o neutra), tipo de foto si tiene, resumen de la nota, palabras clave, presencia virtual de la nota y link a la misma si la tiene.

Esta información fue utilizada para realizar un análisis cualitativo en clave comparada del tratamiento editorial de la cumbre intercoreana por parte de ambos diarios, información que se buscó resumir a partir de una nube de palabras construida en línea. Finalmente se seleccionaron y compararon en profundidad las notas con una valencia explícita (Zunino, 2016) ya que ésta es un proxy de presencia de una línea editorial mayormente marcada para poder visualizar la diferencia en el tratamiento por parte de ambos medios gráficos.

\section{Marco teórico}


¿Miramos el mundo a través de la grieta? Análisis del tratamiento editorial de la cumbre intercoreana desde Argentina

A fin de poder abordar las preguntas que la cumbre de las Coreas nos presenta frente a la relevancia y el tratamiento editorial que los medios de comunicación masivos argentinos le profieren, el trabajo se sirvió de las teorías del Framing, Agenda-setting e Intermedia Agenda-setting.

El Framing, en tanto teoría, explica todo el proceso de la comunicación de los medios masivos. El proceso de encuadre (framing) implica tanto el momento de elaboración de la noticia, como el texto y el contenido de la misma, así como los efectos que ésta pretende tener y efectivamente tiene en la sociedad (Amadeo, 2008).

En primer lugar, se seleccionan los "hechos críticos" a ser reportados, y el ángulo bajo el cual éstos serán analizados. Según explica Pride (citado en Amadeo, 2008, p.188), al ser los acontecimientos re-definibles, son los medios y otros actores sociales de relevancia los que determinan que estos eventos sean críticos (si los cubren y con qué frecuencia), y los definen con la carga valorativa y marcos cognoscibles con los que deciden tratar dicho evento, a priori, objetivo. A través de este marco conceptual se abordará la relevancia sobre la que versa la primera pregunta de esta investigación.

El segundo momento de la teoría, vinculado a la pregunta por las líneas editoriales, es la elaboración de la noticia. Aquí, se pone el foco en los atributos de las noticias como elementos utilizados para transmitir la información y alcanzar los efectos deseados: palabras, recursos retóricos, disposición de la información, presencia o ausencia de fotografía, así como la existencia de valencia -positiva, negativa o ausente mediante una mera descripción-. Las definiciones que entienden que los frames (marcos que hacen a la realidad inteligible) son construcciones que se derivan del contexto simbólico de los periodistas se complementan con aquellas que explican que los mismos se definen de acuerdo con su funcionalidad para los lectores (Amadeo, 2008, p.191).

Adoptar la teoría del Framing implica negar la posibilidad de cualquier postura objetiva por parte del periodista. La objetividad se restringe al respeto de las pautas que dan cuenta que una noticia es producida de forma rigurosa y asequible a la audiencia (Amadeo, 2008, p.217). 
Las teorizaciones que analizan el tercer nivel de framing dan relevancia a los efectos que producen las noticias desde los medios a los emisores (Amadeo, 2008, p.192). Si bien el alcance del presente trabajo no llega a abarcar esta instancia, es menester tenerla en cuenta si se entiende a los medios como actores sociales que influyen en la construcción de la opinión pública.

Se complementará la teoría del Framing con la teoría de la Agenda-setting. La primera de ellas resulta útil a los fines de dar cuenta de la idea que organiza el contenido y que sugiere cuál es el tema para hablar mediante el uso de selección, énfasis, exclusión y elaboración (Tankard citado en Zunino, 2016, p.131). La teoría del Agenda-setting, por su parte, facilita la comprensión de los efectos del contenido en la opinión pública, es por ello que ésta se enfoca en un análisis de contenido (Amadeo, 2008, p.222-224). Sostiene que los medios influyen en determinar sobre qué temas la audiencia debe hablar y la forma en la que estos temas son abordados (Zunino, 2016, p.129).

La forma en que la teoría de la Agenda-setting complementa la del Framing es al momento de contestar la pregunta por la relevancia. En un primer nivel, el recorte de la realidad noticiable, y, en un segundo nivel, la carga valorativa y sustantiva asignada a determinado tema. En este sentido, se retoma a autores como Kiousis (2004), y Manheim (1986), cuya agenda de investigación permite comprender el componente valorativo, sumado a la relevancia. Se rescatan las tres dimensiones de análisis de Kiousis (2004), que dan cuenta de la relevancia en tanto concepto multidimensional: la atención, relacionada con la frecuencia de la cobertura; la prominencia, vinculada con la jerarquía de la información, que se analizó con datos cuantitativos y la valencia o carga valorativa, que se trabajó a partir de información cualitativa (Kiousis, 2004; Zunino, 2016).

La primera dimensión que interesa es la que se refiere a la atención que el medio profiere al acontecimiento y se vincula con la frecuencia en la que el tema es 
¿Miramos el mundo a través de la grieta? Análisis del tratamiento editorial de la cumbre intercoreana desde Argentina

retomado o el espacio dedicado a los títulos. La segunda es la prominencia, ligada al posicionamiento jerárquico del tema en relación a otros (Kiousis, 2004, p.74-75).

La tercera dimensión analizada, el concepto de valencia, será retomado a la hora de responder la segunda pregunta de investigación -referida a las líneas editoriales. La misma remite a los componentes afectivos, el peso emocional que caracteriza al segundo nivel de análisis de la teoría de la Agenda-setting. Da cuenta de los atributos que influyen en la opinión pública y que acompañan los acontecimientos cubiertos en las noticias. Según explica Zunino (2016), la importancia de los atributos radica en que "el modo en el que un tema es cubierto por los medios afecta la forma en que el público piensa acerca del mismo" (Zunino, 2016, p.131). La existencia de un determinado carácter valorativo, sea positivo o negativo, para Kiousis (2004), determina que la noticia tenga mayor importancia.

Por otro lado, se retoman otras teorías que se inscriben dentro de la Agendasetting orientadas a investigar la relación entre los medios. La Intermedia Agendasetting explica que los medios de mayor tirada terminan por condicionar la agenda de otros, como explican McCombs y Bell: "si la prensa establece la agenda pública ¿quién establece la agenda de los medios?” (citado en Jang, 2010, p.14, traducción propia). A los fines de corroborar o refutar la hipótesis, interesa revisitar a los autores enmarcados en esta teoría que corresponden a la segunda "capa" de fuentes de información, que distinguen Casermeiro y Pereson (citado en Aruguete, 2013) y que retoma Aruguete (2015), en la cual interactúan los medios entre sí. De esta forma, Aruguete (2015) explica la homogeneización de los medios como un "proceso arterial" que grafica la influencia de los grandes medios sobre los más pequeños en cuanto a la consonancia, entendida como "la dirección, el sentido de las opiniones existentes sobre un asunto" (Aruguete, 2015, p.113). Si bien este fenómeno se puede deber a los mecanismos de socialización comunes entre los reporteros (McCombs y Shaw citado en Aruguete, 2015, p.120), a que un medio o agencia sirva como fuente a diferentes periódicos (Jang, 2010; Lee, Lancendorfer y Lee citado en Jang, 2010, p.14), o al liderazgo ejercido por 
ciertas organizaciones mediáticas (Reese y Danielian citado en Jang, 2010, p.14), en la hipótesis se argumenta que la "estandarización" que se espera encontrar se debe a una falta de interés estratégico directo en el suceso noticiable y que, si bien hay una presión por comunicar el hecho por tener cierta relevancia internacional, su tratamiento no será marcadamente diferenciado.

De esta forma, tanto las teorías del Framing como las de Agenda-setting permitirán develar la relevancia y la valoración que los periódicos le atribuyeron a la cumbre de las Coreas, así como el tratamiento editorial de cada uno. A su vez, la teoría de la Intermedia Agenda-setting sustentaría la hipótesis de este trabajo que apunta a no encontrar diferencias sustanciales entre el tratamiento de ambos medios, al no tratarse de un evento que sea clave para la Argentina. Debido a ello, se daría un proceso de homogeneización, posiblemente replicando perspectivas de periódicos internacionales.

\section{Análisis}

En cuanto a la atención y la jerarquía -primeros dos aspectos de la relevancia, según Kiousis (2004)-, es posible, en un primer momento, afirmar que ésta ha sido alta. Esto se sustenta en el hecho de que, para los quince días analizados, se hayan publicado 21 (veintiún) notas entre ambos diarios. Éstas se concentraron principalmente en el diario Clarín -quince-, mientras que las noticias internacionales de Página 12 se focalizaron más en cuestiones regionales, como el encarcelamiento de Luiz Inácio Lula Da Silva, y la visita de Dilma Rousseff a la Argentina, apareciendo 6 (seis) publicaciones sobre la cumbre intercoreana. No obstante, el periódico Clarín posee una extensión de casi el doble que Página 12.

En cuanto a los días donde se concentraron las noticias sobre la cumbre intercoreana, es notable que la mayoría de ellas (cinco) aparecieron el sábado 28 de abril de 2018, día siguiente a la reunión. Esta cobertura había comenzado en los tres números posteriores. 
¿Miramos el mundo a través de la grieta? Análisis del tratamiento editorial de la cumbre intercoreana desde Argentina

A los efectos de sintetizar la relevancia, los resultados del índice indican que, en promedio, la importancia dada por ambos periódicos es media, con un promedio de 10.33. Para el caso de Clarín, cuyas notas obtienen, en media, un puntaje de 9.93 en nuestro índice, la importancia asignada a la cumbre, es media. Página 12, que obtuvo un valor de 11.33 según la misma herramienta, le dio una importancia media-superior. Así, aunque Clarín haya publicado más contenido sobre el tema, las notas de Página 12 son mayores en su extensión (tanto en cantidad de palabras, como en superficie), y un mayor porcentaje de éstas están firmadas por un especialista.

Tabla 3. Categorías del índice construido

\begin{tabular}{|c|c|c|c|c|c|}
\hline Clasificación & Subdivisión & Puntaje & $\begin{array}{l}\text { Total } \\
\text { notas } \\
\end{array}$ & $\begin{array}{l}\text { Cantidad } \\
\text { de Clarín }\end{array}$ & $\begin{array}{c}\text { Cantidad de } \\
\text { Página } 12\end{array}$ \\
\hline \multirow{6}{*}{ BAJA } & \multirow{2}{*}{ Baja inferior } & 1 & 0 & 0 & 0 \\
\hline & & 2 & 0 & 0 & 0 \\
\hline & \multirow{2}{*}{ Baja } & 3 & 0 & 0 & 0 \\
\hline & & 4 & 1 & 1 & 0 \\
\hline & \multirow{2}{*}{ Baja superior } & 5 & 2 & 1 & 1 \\
\hline & & 6 & 2 & 2 & 0 \\
\hline \multirow{6}{*}{ MEDIA } & \multirow{2}{*}{ Media inferior } & 7 & 0 & 0 & 0 \\
\hline & & 8 & 3 & 3 & 0 \\
\hline & \multirow{2}{*}{ Media } & 9 & 1 & 1 & 0 \\
\hline & & 10 & 1 & 0 & 1 \\
\hline & \multirow{2}{*}{ Media superior } & 11 & 3 & 2 & 1 \\
\hline & & 12 & 0 & 0 & 0 \\
\hline \multirow{6}{*}{ ALTA } & \multirow{2}{*}{ Alta inferior } & 13 & 1 & 0 & 1 \\
\hline & & 14 & 3 & 2 & 1 \\
\hline & \multirow{2}{*}{ Alta } & 15 & 4 & 3 & 1 \\
\hline & & 16 & 0 & 0 & 0 \\
\hline & \multirow{2}{*}{ Alta superior } & 17 & 0 & 0 & 0 \\
\hline & & 18 & 0 & 0 & 0 \\
\hline
\end{tabular}

Fuente: Elaboración propia

No obstante, la moda del conjunto de las notas analizadas presenta una relevancia alta. Esto se debe a la extensión de estas, tanto en cuanto a tamaño por superficie y por cantidad de palabras, a la presencia de grandes titulares, de bajada, de 
elementos gráficos, la publicación en días de fin de semana, y la presencia de una valencia explícitamente definida por parte de la mayoría de las noticias relevadas.

Al ver que la relevancia dada es promedio por ambos periódicos, se puede afirmar, desde el primer momento de la teoría del Framing que si bien la cumbre de las Coreas, a priori, es un hecho internacionalmente relevante de manera que es material noticiable, los medios de comunicación argentinos relevados no lo han constituido con una relevancia alta, aunque lo reconocieron como un "hecho crítico".

En relación con la segunda pregunta de investigación, se procedió a analizar, en primer lugar, la valencia de las notas publicadas por ambos diarios. En una segunda instancia, se realizó una nube de palabras por cada periódico, con base en los términos clave. Por último, se leyó en profundidad del contenido de las piezas periodísticas.

Las publicaciones de Clarín presentan la siguiente distribución en cuanto a su valencia: 7 (siete) de carácter negativo, 5 (cinco) neutrales y 3 (tres) positivas. Esto denota la presencia de una línea editorial por parte del periódico, donde se resaltan las características anti-democráticas, anti-capitalistas y extorsivas de Corea del Norte. Estas características no siempre son marcadas de manera explícita, sino que también recurren a la construcción de frames. Por ejemplo, en la nota 15.2, se recorta la realidad reportada en términos de información en cuanto a los efectos de los ensayos nucleares y los testimonios relevados, como geográficamente en cuanto a la ubicación de la planta nuclear norcoreana y las regiones chinas que ésta afectaría. Asimismo, hay una incidencia de los frames en un segundo momento en cuanto a la elección de determinados atributos para utilizar en el cuerpo del texto, al emplear palabras como "régimen comunista" o apelando a la emocionalidad de los lectores invocando imágenes sensibles como los "chicos de escuelas primarias" que aparecen afectados. Otro caso de este tipo es la nota 11.2, donde se presenta una fotografía del "ensayo de uno de los más potentes misiles del régimen", sin mostrarse una imagen de los despliegues militares de Estados Unidos en la península como contraparte. 
¿Miramos el mundo a través de la grieta? Análisis del tratamiento editorial de la cumbre intercoreana desde Argentina

Para el caso de Página 12, 3 (tres) de las notas tienen una valencia neutral y 3 (tres) una valencia positiva. La nota 12.1 celebra el cónclave, pero no por ello deja de destacar que no se establecieron medidas específicas más allá de las promesas, y también resalta las posturas “en reserva” de distintos líderes mundiales. La 18.1, escrita por Atilio Borón, ve con buenos ojos la cumbre porque desviaría la atención de Estados Unidos desde Cuba hacia Corea del Norte, humillando así a la Casa Blanca. De esta forma se vuelve a notar los frames utilizados en tanto recorte y enfoque por parte de Página 12 que remite mayormente a cuestiones regionales aún al analizar conflictos lejanos.

Como se desprende de las nubes de palabras, el tema privilegiado por ambos periódicos en relación a la cumbre intercoreana es el tema de la desnuclearización de la península coreana.

Ahora bien, en una segunda lectura, los periódicos enfatizan asuntos diferentes. Mientras que en Clarín los términos utilizados más frecuentemente para referirse a la cumbre son "líder" y "régimen"; en Página 12 resaltan "guerra" y “distensión".

En el caso de Clarín, "líder" es el término más frecuentemente utilizado para referirse a Kim Jong-un, acompañado generalmente de la calificación de "comunista". Esto se opone a la forma en que alude a Moon Jae-In, a quién lo nombra, en la nota 11.2, como "presidente socialdemócrata". Asimismo, el término "régimen", por oposición a palabras como "gobierno", suele estar acompañado de calificativos como "pseudo comunista". También se alude al régimen como "dictadura comunista" y se habla de la "capacidad coercitiva del régimen”. Si bien Clarín se ocupa más de castigar a Corea del Norte, dos de las notas analizadas resaltan la doble acepción posible del término "desnuclearización": la destrucción del arsenal nuclear norcoreano y/o la retirada de las tropas norteamericanas de la península.

Por su parte, Página 12 incluye directamente a Estados Unidos en su análisis, mediante de la mención a Donald Trump, la Agencia Central de Inteligencia (CIA) y 
Washington. Esto indica que, a la hora de interpretar la cumbre, lo hace en términos acentuadamente geopolíticos. Esto también se ve al mencionar a Cuba. Por otra parte, destacan palabras positivas acerca de la reunión, aunque de una manera cautelosa.

Por otro lado, el $60 \%$ de las notas publicadas en Clarín son de agencias internacionales de noticias, $20 \%$ con firmas de autor y $20 \%$ atribuibles a la editorial.

El primer porcentaje mencionado permite hacer uso de la teoría del Intermedia Agenda-setting al reconocer la interacción llevada adelante entre los medios y la incidencia de las agencias de mayor alcance en la definición de los temas a tratar y la forma en la que estos son abordados. Al no existir influencia alguna por parte de agencias internacionales en Página 12, no se puede hablar de una homogeneización entre ambos medios en el establecimiento y tratamiento de la agenda, explicado por este factor.

En cuanto al contenido de Clarín, destacan tres grandes líneas argumentales: la afirmación de que Corea del Norte sólo accedió a negociar con Corea del Sur y Estados Unidos una vez que fue una potencia nuclear; la influencia y la opinión de las potencias mundiales en y acerca del acuerdo; y la celebración, a pesar de estas reservas, de la cumbre.

En el primer grupo de argumentos, se destacan las notas 11.1; 11.2 13.1, y 25.1, que recalcan la actitud extorsiva y disuasiva de Corea del Norte ahora que tiene capacidad nuclear para destruir cualquier capital del mundo, y la desconfianza que eso genera en Estados Unidos y Corea del Sur, país que, según el medio (13.1), habría hecho menos propaganda sobre la cumbre que su contraparte del norte. En el mismo sentido, Clarín publicó el siguiente cómic un día después de la cumbre.

Figura 1. "Tira y afloja" 
¿Miramos el mundo a través de la grieta? Análisis del tratamiento editorial de la cumbre intercoreana desde Argentina

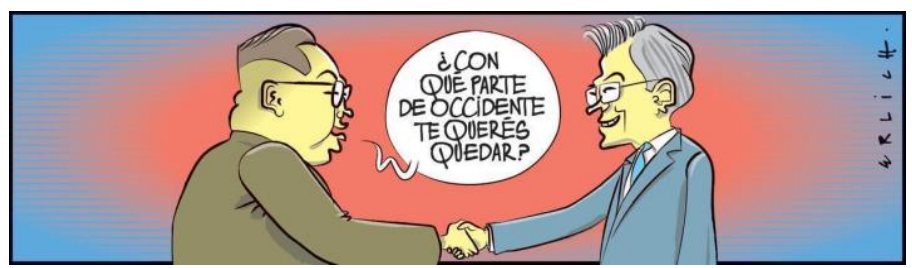

Fuente: Erlich, 2018 (nota 11.4, 28/04/2018)

Éste resalta el tono de desconfianza con el que el multimedio presenta la cumbre, especialmente centrado en el líder norcoreano, que es quien habla.

El segundo grupo de argumentos, compuesto por las notas $11.1 ; 11.2 ; 13.2 \mathrm{y}$ 27.1, permite ver que, en un análisis más pormenorizado, Clarín realiza también un abordaje geopolítico de la cuestión: alerta sobre la posibilidad de que Trump no negocie satisfactoriamente con Corea del Norte, y que ésta pueda doblegarlo, así como las miradas desconfiadas de Japón y Corea del Sur sobre las intenciones de Kim. Por otro lado, destaca que "el gigante chino" (1.1) instruye a los diplomáticos norcoreanos.

En tercer lugar, aparecen menciones positivas a la reunión en las notas 11.2; $13.1 ; 15.2 ; 17.1 ; 21.1$ y 25.1. Éstas aluden a la decisión de formar un equipo conjunto de Ping Pong por parte de ambas Coreas, a la distensión, al enfriamiento de las relaciones, a la desnuclearización de la península, a la "paz perpetua" (15.1), y a la "buena onda" (11.2).

Por parte de Página 12 las notas relevadas acusan un tono entre neutral y positivo. Este periódico tiene un enfoque que privilegia los factores geopolíticos. Por ejemplo, en la nota 10.1, escrita por Julián Varsavsky, el autor afirma: "Paradójicamente, el último capítulo de la Guerra Fría del siglo XX, se sigue peleando en la península coreana". Asimismo, se alude a la cumbre en un tono que, si bien es positivo, es también cauteloso ante la reserva por parte de los líderes mundiales. Este es el caso de la nota 12.1, donde se resaltan palabras como "optimismo" y, “acercamiento", pero también "cautela". En lo referente a la imagen de ésta, se muestra simbólicamente positiva hacia una cooperación en conjunto. El título, “Todos felices 
con la cumbre de las Coreas", sigue esta misma línea, disonando con el tono crítico del cuerpo de la nota.

\section{Conclusiones}

Sobre la primera pregunta, referida a la relevancia, es posible concluir que el promedio de ésta fue medio, no obstante, la moda fue alta. La frecuencia por parte de Clarín de cobertura de la cumbre intercoreana fue superior a la de Página 12. Mientras que el multimedio cubrió el hecho con quince notas, el segundo lo hizo con seis. Sin embargo, al cuantificar la importancia en el índice, el promedio atribuido a Página 12 fue superior frente al de Clarín.

En relación con la segunda pregunta, sobre el tratamiento editorial, ambos periódicos pusieron como tema central a la desnuclearización de la península coreana en sentido amplio. Si bien los dos periódicos hacen un análisis en clave geopolítica en términos globales, Página 12 también hace hincapié en las consecuencias de dicho encuentro con respecto a la región de América Latina, al introducir la cuestión cubana. Con respecto a la valencia con la que ambos medios gráficos han tratado el hecho, ésta sí fue diferenciada. Mientras que Clarín ha tenido un tratamiento más bien negativo, en 7 (siete) de 15 (quince) notas, en Página 12 no hay notas de carácter negativo.

En cuanto al posible fenómeno de homogeneización (Intermedia Agendasetting), no es posible corroborarlo. Si bien en Clarín se encontró una gran cantidad de notas extraídas de agencias, esto no sucedió en Página 12. Mientras que en el primer caso podría llegar a haber un efecto de Intermedia Agenda-setting, en el segundo no, o al menos no de manera empíricamente observable, ya que el tratamiento ha sido exclusivamente a través de su propia editorial o expertos en el tema.

En suma, las presunciones iniciales se cumplen sólo parcialmente. En cuanto a la relevancia, la falta de vínculo estratégico entre Argentina y las Coreas no impidió la aparición de notas en tapa y contratapa en ambos periódicos. Sin embargo, tampoco 
¿Miramos el mundo a través de la grieta? Análisis del tratamiento editorial de la cumbre intercoreana desde Argentina

se puede afirmar que la importancia dada al hecho haya sido alta, sino media. Con relación al tratamiento editorial, la hipótesis se corrobora en una primera lectura, ya que ambos medios se enfocaron en la desnuclearización de la península coreana y sus consecuencias geopolíticas. No obstante, en una segunda lectura, el diario Clarín tomó un posicionamiento notoriamente favorable hacia Corea del Sur, reconociéndole la iniciativa del encuentro, y contrario a Corea del Norte, sin escatimar golpes retóricos a su líder y gobierno.

Del presente trabajo se desprende una conclusión general sobre las características de los medios de comunicación en contextos políticos polarizados, como el argentino. Se observa que el fenómeno de "la grieta" no permea solamente los eventos políticos nacionales o regionales, sino que se aplica también a la lectura de hechos geográficamente lejanos, y hasta políticamente poco relevantes para la Argentina, como ser la cumbre intercoreana.

\section{Referencias bibliográficas}

Amadeo, Belén (2008). Framing: Modelo para armar. EDUCA. Argentina. (Pp.183237).

Aruguete, Natalia y Amadeo, Belén (2013). Encuadrando el delito: pánico moral en los periódicos argentinos. América Latina Hoy, Revista de Ciencias Sociales, Universidad de Salamanca. No. 62, (Pp. 177-196).

Aruguete, Natalia (2015). El poder de la agenda. Biblos. Argentina, (Pp. 105-124 y 159-174).

Clarín (2018). Las dos Coreas acuerdan profundizar los vínculos y desnuclearizar la península. 28 de abril de 2018. Consulta: http://bit.ly/Clarín-Las2Coreas

Erlich, Leandro (2018). Tira y afloja [Ilustración]. Clarín. 28 de abril de 2018. Argentina.

Gallart, María Antonia (1992). La integración de métodos y la metodología cualitativa. Una reflexión desde la práctica de la investigación. En F. Forni y otros. Métodos cualitativos II. La práctica de la investigación. CEAL. Argentina. Instituto Nacional de Estadísticas y Censos (INDEC) (2018). Intercambio comercial argentino: cifras estimadas de diciembre de 2017. Extraído de: https://www.indec.gob.ar/uploads/informesdeprensa/ica_01_18.pdf Consulta: $10 / 05 / 18$.

Instituto Verificador de Circulaciones (IVC) (2018). El Boletín Xpress Instituto Verificador de Circulaciones. Febrero 2018. Argentina (Pp. 12-16). 
Jang, Seckjun (2010). Intermedia agenda setting effects between internet bulletin boards and traditional news media in U.S. and Korean presidential campaigns. Tesis Doctoral. University of Texas at Austin. Austin, Texas, United States.

Kiousis, Spiro (2004). Explicating Media Salience: A Factor Analysis of New York Times Issue Coverage during the 2000 U.S. Presidential Election. Journal of Communication. Volumen 54 No. 1. Estados Unidos. (Pp. 71-87).

Manheim, Jarol B. (1986). A model of agenda dynamics. Communication Yearbook No. 10. Estados Unidos. (Pp. 499-516).

Martini, Stella y Luchessi, Lila (2004). Periodismo, información y poder. Biblos. Argentina.

Oviedo Eduardo Daniel (2016). Política y económica en las relaciones argentinocoreanas. Miríada. No. 12. Argentina. (Pp.119-149).

Zunino, Esteban (2016). La relevancia de las noticias en la prensa gráfica. Una reflexión teórico-metodológica a partir del análisis del conflicto entre las corporaciones agrarias y el gobierno argentino. Nueva época. Universidad de Guadalajara. No. 25. México. (Pp. 127-156). Extraído de: http://www.scielo.org.mx/pdf/comso/n25/n25a6.pdf. Consulta: 05/05/18. 